\title{
Effects of bodyweight support and guidance force on muscle activation during Locomat walking in people with stroke: a cross-sectional study
}

\author{
Jianhua Lin ${ }^{1,2^{*}}$, Guojiong Hu', Jun Ran' ${ }^{1}$ Linyu Chen ${ }^{1}$, Xian Zhang ${ }^{1}$ and Yanxin Zhang ${ }^{3}$
}

\begin{abstract}
Background: Locomat is a robotic exoskeleton providing guidance force and bodyweight support to facilitate intensive walking training for people with stroke. Although the Locomat has been reported to be effective in improving walking performance, the effects of training parameters on the neuromuscular control remain unclear. This study aimed to compare the muscle activities between Locomat walking and treadmill walking at a normal speed, as well as to investigate the effects of varying bodyweight support and guidance force on muscle activation patterns during Locomat walking in people with stroke.

Methods: A cross-sectional study design was employed. Participants first performed an unrestrained walking on a treadmill and then walked in the Locomat with different levels of bodyweight support (30\% or 50\%) and guidance force $(40 \%$ or $70 \%)$ at the same speed $(1.2 \mathrm{~m} / \mathrm{s})$. Surface electromyography (sEMG) of seven muscles of the affected leg was recorded. The sEMG envelope was time-normalised and averaged over gait cycles. Mean sEMG amplitude was then calculated by normalising the sEMG amplitude with respect to the peak amplitude during treadmill walking for statistical analysis. A series of Non-parametric test and post hoc analysis were performed with a significance level of 0.05 .

Results: Fourteen participants with stroke were recruited at the Yangzhi Affiliated Rehabilitation Hospital of Tongji University (female $n=1$; mean age $46.1 \pm 11.1$ years). Only the mean sEMG amplitude of vastus medialis oblique during Locomat walking (50\% bodyweight support and 70\% guidance force) was significantly lower than that during treadmill walking. Reducing both bodyweight and guidance increased muscle activity of gluteus medius and tibialis anterior. Activity of vastus medialis oblique muscle increased as bodyweight support reduced, while that of rectus femoris increased as guidance force decreased.

Conclusions: The effects of Locomat on reducing muscle activity in people with stroke were minimized when walking at a normal speed. Reducing bodyweight support and guidance force increased the activity of specific muscles during Locomat walking. Effects of bodyweight support, guidance force and speed should be taken into account when developing individualized Locomat training protocols for clients with stroke.
\end{abstract}

Keywords: Stroke, Locomat, Gait, Electromyography, Rehabilitation

\footnotetext{
* Correspondence: rexjhlin@163.com

'Department of Rehabilitation Therapy, Yangzhi Affiliated Rehabilitation

Hospital of Tongji University, No. 2209, Guangxing Road, Songjiang District,

Shanghai 201619, People's Republic of China

${ }^{2}$ Faculty of Health Sciences, The University of Sydney, Sydney, New South

Wales, Australia

Full list of author information is available at the end of the article
}

(c) The Author(s). 2020 Open Access This article is distributed under the terms of the Creative Commons Attribution 4.0 International License (http://creativecommons.org/licenses/by/4.0/), which permits unrestricted use, distribution, and

reproduction in any medium, provided you give appropriate credit to the original author(s) and the source, provide a link to the Creative Commons license, and indicate if changes were made. The Creative Commons Public Domain Dedication waiver (http://creativecommons.org/publicdomain/zero/1.0/) applies to the data made available in this article, unless otherwise stated. 


\section{Introduction}

Gait disturbance is one of the major consequences associated with stroke. Due to the impaired supraspinal control, the gait pattern post stroke is characterized as muscle weakness, spasticity, abnormal muscular amplitude and asymmetrical temporal ordering of muscle activity $[1,2]$. Impaired walking ability not only reduces the functional independency of stroke survivors, but also increases a series of risks, like fall [3-5]. The restoration of functional walking ability requires intensive training with a symmetrical gait pattern [6-8].

Various robot-assisted gait trainers, like Locomat, G-EO system Evolution and Gait Trainer, have been designed and implemented in gait rehabilitation for stroke patients [9-15]. These gait trainers enable a repetitive walking training with predefined normal gait pattern and largely reduce the physical demand of therapists [16]. Those robot-assisted gait trainers, like Locomat (Hocoma, Switzerland), can provide a range of adjustable functions, including bodyweight support (BWS), guidance force (GF) and walking speed, allowing clinicians to develop an individualised training protocol that best fits patient's ability level $[17,18]$. Locomat training, however, has been found to reduce muscle activities in both healthy individuals and people with stroke when compared to overground walking $[19,20]$. For example, Coenen and colleagues [20] found that the application of BWS and GF significantly reduced activities of several muscles of affected leg in people with stroke. This feature of Locomat training is considered as a negative aspect of its clinical implication because voluntary contraction of muscles plays a key role in motor relearning [21]. In addition, the exoskeletons of Locomat limit the movement in sagittal plane and reduce the degree of freedom of pelvis which may lead to abnormal interaction between the leg and exoskeleton as well as abnormal muscle activity pattern [10, 22].

There is sufficient evidence showing that the Locomat training provided better improvement in terms of independent walking ability, walking speed, balance and disability than conventional physiotherapy to people with stroke [23-28]. There is also evidence that Locomat training significantly improved the duration of single stance phase, step length ratio on the paretic leg when walking on the ground $[29,30]$. However, there are also studies showing that the Locomat was not superior to conventional therapy in people with stroke [9, 30, 31]. Despite the heterogeneous features of participants, the difference in training parameters of Locomat may also contribute to the controversial results. In healthy participants, there is ample evidence that BWS or GF can affect the activation of specific muscles $[10,19,20,32$, 33]. There are also studies reporting significant interactions between BWS, GF and walking speed on voluntary control indicating that the mechanisms of those parameters are complex [32]. In a recent study, however, researchers reported that varying BWS and GF was not associated with changes of muscle activity in people with stroke, whereas increasing walk speed led to greater muscle activity [34]. Since the walking speeds used in previous studies were relatively low $(0.56 \mathrm{~m} / \mathrm{s}$ and 0.61 $\mathrm{m} / \mathrm{s}$ respectively) $[19,20]$ and the increase of speed was associated with greater muscle activity [32, 35], it is of interest to investigate whether a higher walking speed would modulate the difference in muscle activity between Locomat walking and treadmill walking.

To further investigate the effects of BWS and GF on active muscle activity, this study aimed to compare the muscle activity level of affected leg between Locomat and treadmill walking at a normal speed in people with stroke. This study also investigated the effects of varying BWS and GF on muscle activity patterns during Locomat walking. Therefore, we hypothesized that when walking at a normal speed, people with stroke exhibit lower muscle activity in the affected leg during Locomat walking than during unrestrained treadmill walking. We also hypothesized that reducing BWS and GF will increase muscle activity level of the affected leg in people with stroke.

\section{Methods}

This was a cross-sectional study which compared the muscle activity of affected leg among different walking conditions in people with stroke. Participants were recruited from inpatients that were receiving rehabilitation at Shanghai Yangzhi Affiliated Rehabilitation Hospital of Tongji University between 13 July 2017 and 29 June 2018. A senior physiotherapist (GJH) was responsible for eligibility screening. Participants were eligible for this study if they were post stroke, aged 18 years or above, had abnormal gait but could walk independently at 1.2 $\mathrm{m} / \mathrm{s}$ without assistance, and had no Locomat training experience. The abnormal gait in present study refers to the gait that is characterized by compensative movement pattern due to reduced selective motor control (like leg circumduction, pelvic hiking, drop foot during swing phase), asymmetrical spatial and temporal performance (like reduced stride length, shortened single stance time of affected leg and increased step width). Participants were excluded if they had trauma or surgical history in lower limb, severe osteoporosis, cognitive impairment and pathological complications, or had pain during walking. Written informed consents were obtained from all participants before testing.

\section{Experimental protocols}

Prior to trials, detailed instruction and explanation of the experiment was provided to each participant. Participants were first asked to complete a treadmill walking 
trial without exoskeleton. Subsequently, they performed four walking trials with Locomat under different combinations of BWS $(30 \%$ or $50 \%$ of participant's body weight) and GF ( $40 \%$ or $70 \%$ ) in a randomized order (see Table 1). The walking speed for all trials was set at $1.2 \mathrm{~m} / \mathrm{s}$. For each trial, participants walked for $3 \mathrm{~min}$. The first 2 min walking served as warm up allowing participants to get used to the walking condition while the last minute walking was used for analysis. Participants were allowed to take an at least 3-min break between two trials. Elastic foot lifter was applied to all participants during walking in the Locomat. The movement of affected leg was recorded for all walking conditions (frame rate $=30 \mathrm{fps})$.

\section{Surface electromyography recording and analysis}

Muscle activities of affected leg during walking were recorded by using surface electromyography (sEMG) (Noraxon U.S.A., Inc.) from gluteus medius (GM), vastus medialis oblique (VMO), vastus lateralis oblique (VLO), rectus femoris $(\mathrm{RF})$, biceps femoris (BF), medial gastrocnemius (MG) and tibialis anterior (TA) (see Fig. 1). Prior to the placement of electrodes, the body hair in the electrode sites was shaved and then the skin was abraded and cleaned [36]. The disposable $\mathrm{Ag} / \mathrm{AgCl}$ electrodes (272S, Noraxon USA, Inc., Scottsdale, AZ, USA) with a $10 \mathrm{~mm}$ diameter and a $20 \mathrm{~mm}$ inter electrode distance were placed parallel to the muscle fibre according to the SENIAM recommendations [37]. The sEMG sampling frequency was set at $1500 \mathrm{~Hz}$. The sEMG signal was synchronized with the video of walking trials.

The raw sEMG signal was first processed by a $20 \mathrm{~Hz}$ second-order high-pass Butterworth filter and then rectified. A $4 \mathrm{~Hz}$ fourth-order low-pass Butterworth filter was applied afterwards. Then the root-mean-square envelope of the sEMG signal was calculated using a moving window $(100 \mathrm{~ms})$. The amplitude of sEMG envelope under each walking condition was normalised with respect to the peak amplitude during unrestrained treadmill walking [34].

The gait cycle was defined as the duration between two consecutive heel strikes [19]. The heel strikes were detected by manual inspection of the video of walking trials. The sEMG data of each gait cycle was time normalized into 100 data points. The mean sEMG amplitude of the gait cycle was calculated as the mean value of the 100 data points and averaged over the gait cycles

Table 1 Walking conditions of Locomat trials

\begin{tabular}{lll}
\hline Condition & Bodyweight support & Guidance force \\
\hline 1 & $50 \%$ & $70 \%$ \\
2 & $50 \%$ & $40 \%$ \\
3 & $30 \%$ & $70 \%$ \\
4 & $30 \%$ & $40 \%$ \\
\hline
\end{tabular}

for each muscle and each participant. The averaged mean sEMG amplitude was used for statistical analysis. A figure of sEMG profiles over a gait cycle was created to display the averaged muscle activity pattern of each muscle under each walking condition (see Fig. 2).

\section{Statistical analysis}

Mean and standard deviation were calculated for age, course post stroke and mean sEMG amplitude. The mean sEMG amplitudes under all five conditions were compared by using Friedman test as the sphericity assumption for repeated measures ANOVA was violated for all muscles. Post hoc analysis was performed by using Wilcoxon signed-rank test with Bonferroni correction for multiple comparisons. The statistical analysis was performed with IBM SPSS Statistics 22. The significance level for Friedman test was set at 0.05 and the significance level for post hoc test was set at 0.005 .

\section{Results}

Fourteen participants were recruited for this study. The demographic data are shown in Table 2. The averaged sEMG pattern over a gait cycle is presented in Fig. 2. The mean sEMG amplitudes of each muscle under all walking conditions and their comparisons are shown in Fig. 3. Although significant within-subject changes were revealed by Friedman test for all muscles, only a few significant changes were showed by post hoc analysis.

\section{Comparison between Locomat walking and unrestrained treadmill walking} Most muscles exhibited similar activation patterns during Locomat walking to that during treadmill walking except BF, RF and TA (see Fig. 2). The activation level of BF during the first half of gait cycle during Locomat walking was remarkably higher than that during treadmill walking. An obvious increase of activation level was also found in RF and TA during the latter half of gait cycle during Locomat walking.

Most muscles demonstrated lower mean sEMG amplitudes during Locomat walking (except under 30\% BWS and $40 \% \mathrm{GF}$ ) than unrestrained treadmill walking, except the GM and BF (see Figs. 2 and 3). However, only the difference in mean sEMG amplitude of VMO between Locomat walking (with 50\% BWS and 70\% GF) and unrestrained treadmill walking was statistically significant (see Fig. 3).

\section{Effects of varying bodyweight support and guidance force during Locomat walking}

In general, the mean sEMG amplitude of muscles tended to increase as the BWS and GF decreased (see Figs. 2 and 3). GM and TA demonstrated significant increases of mean sEMG amplitude when BWS and GF decreased 


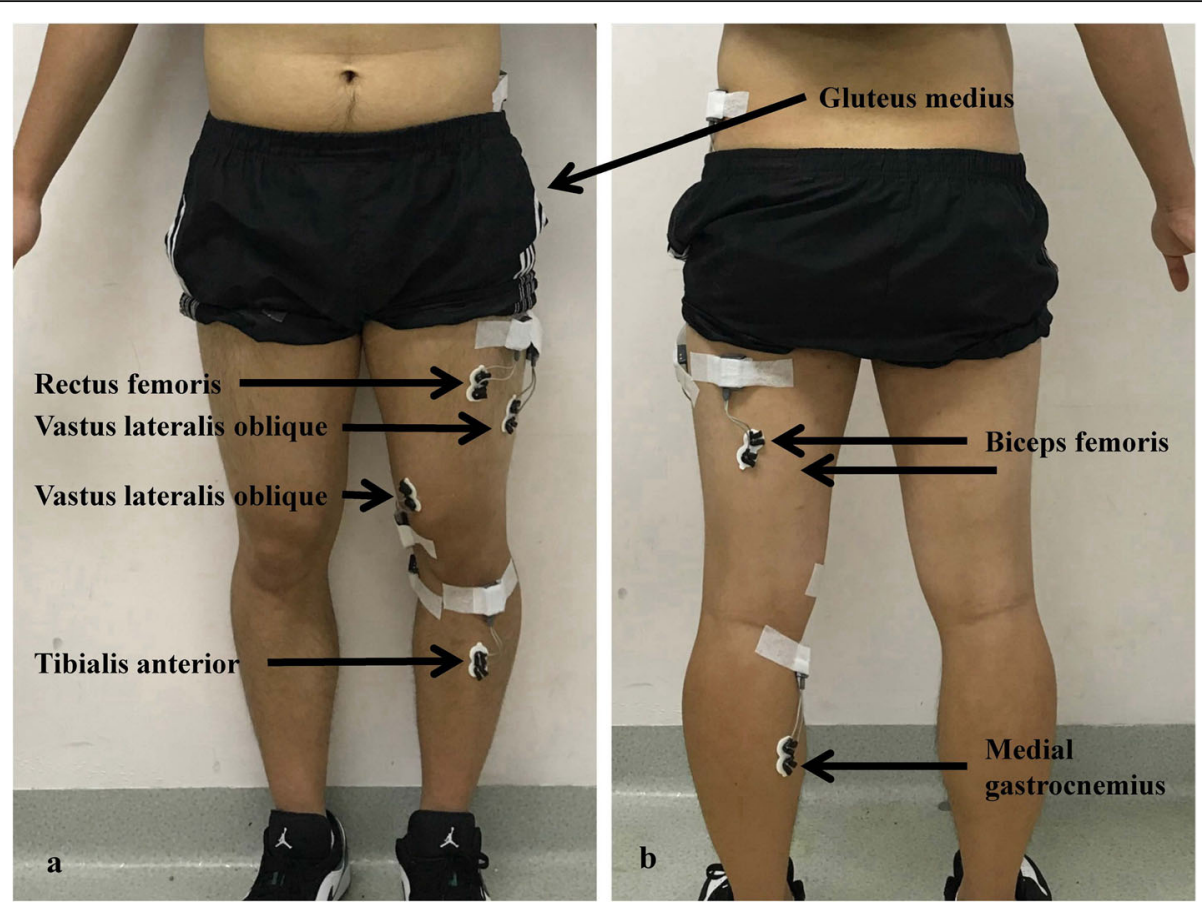

Fig. 1 Placement of electrodes. a: the front view; $\mathbf{b}$ : the back view

from 50 to $30 \%$ and from 70 to $40 \%$ respectively. When GF was at $40 \%$, mean sEMG amplitude of VMO increased significantly as BWS decreased from 50 to $30 \%$. When BWS was at $30 \%$, reduction of GF was associated with a significant increase of mean sEMG amplitude in RF. No other significant change was found for the rest muscles.

\section{Discussion}

The present study found that the application of BWS and GF during Locomat walking only reduced the muscle activity in VMO compared to unrestrained treadmill walking in people with stroke when a normal speed was selected. The results of this study also showed that reducing BWS and GF led to increased muscle activities in GM, VMO RF and TA.

\section{Locomat walking vs. unrestrained treadmill walking}

Although Locomat walking generally tended to reduce the mean activity level in most of the muscles, the present study showed that it only significantly reduced the activity level of VMO compared to unrestrained treadmill walking when a normal walking speed was selected. This finding was inconsistent with previous studies where significant reduction of muscle activity was found in GM, VLO, RF, MG and TA in people with stroke $[19,20]$. Since the settings of BWS and GF in present study were similar to their studies, the different results may be attributing to the different walking speeds between studies. The walking speed in their studies were $0.56 \mathrm{~m} / \mathrm{s}$ and $0.61 \mathrm{~m} / \mathrm{s}[19,20]$, which were much lower than that in our study $(1.2 \mathrm{~m} / \mathrm{s})$. Sufficient evidence has shown that higher walking speed was associated with greater muscle activity as to meet the higher demand of energy output [34, 35, 38-41]. The different findings between studies may suggest that the effects of Locomat training on reducing muscle activity in people with stroke may be eliminated by using a normal walking speed.

This study also found that GM and BF showed higher mean sEMG amplitudes during Locomat walking, although the differences were not statistically significant. In theory, offering BWS and GF during Locomat walking could facilitate movement control of legs by reducing muscle load. However, the effects of Locomat walking on muscle activity are inconsistent in the literature. Some studies have shown that Locomat walking was associated with lower muscle activity compared to treadmill walking in both healthy individuals and people with stroke $[19,20]$, while other studies have found that Locomat walking increased muscle activity in several leg muscles, including BF, quadriceps and gluteus muscles in heathy participants $[10,42]$. As suggested in previous studies, the increase of muscle activity may be the results of interaction between the active leg movement and the inertia of the exoskeleton [10, 43]. During Locomat walking, the built-in gait patterns according to which the exoskeletons drive leg movements may restrict the active leg movement in sagittal plane and the pelvic movement 


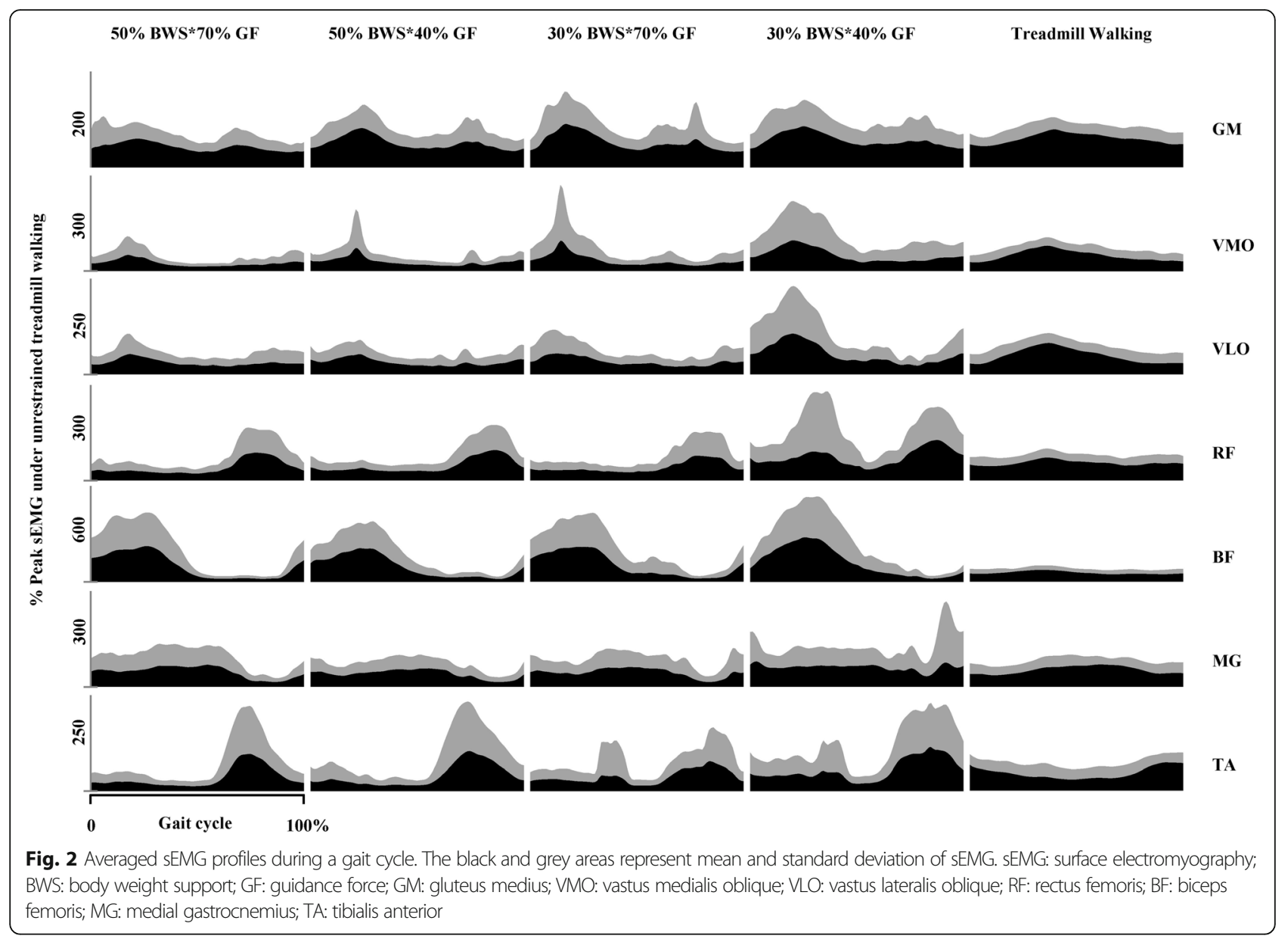

Table 2 Demographic characteristic of participants

\begin{tabular}{|c|c|c|c|c|c|}
\hline Participant number & Gender & Age (years) & Affected side & Stroke type & Time since stroke (months) \\
\hline 1 & Male & 36 & Left & Infarction & 1.5 \\
\hline 2 & Male & 32 & Right & Haemorrhage & 9.3 \\
\hline 3 & Male & 59 & Left & Infarction & 2.1 \\
\hline 4 & Male & 36 & Right & Infarction & 1.2 \\
\hline 5 & Male & 52 & Right & Infarction & 12.1 \\
\hline 6 & Female & 69 & Left & Infarction & 1.6 \\
\hline 7 & Male & 33 & Left & Infarction & 2.5 \\
\hline 8 & Male & 38 & Right & Haemorrhage & 6.7 \\
\hline 9 & Male & 35 & Right & Infarction & 1.9 \\
\hline 10 & Male & 57 & Left & Infarction & 10.2 \\
\hline 11 & Male & 45 & Left & Haemorrhage & 3.6 \\
\hline 12 & Male & 52 & Left & Infarction & 31.4 \\
\hline 13 & Male & 52 & Left & Infarction & 5.7 \\
\hline 14 & Male & 52 & Right & Haemorrhage & 8.9 \\
\hline Mean \pm SD & & $46.29 \pm 11.48$ & & & $7.05 \pm 7.93$ \\
\hline
\end{tabular}




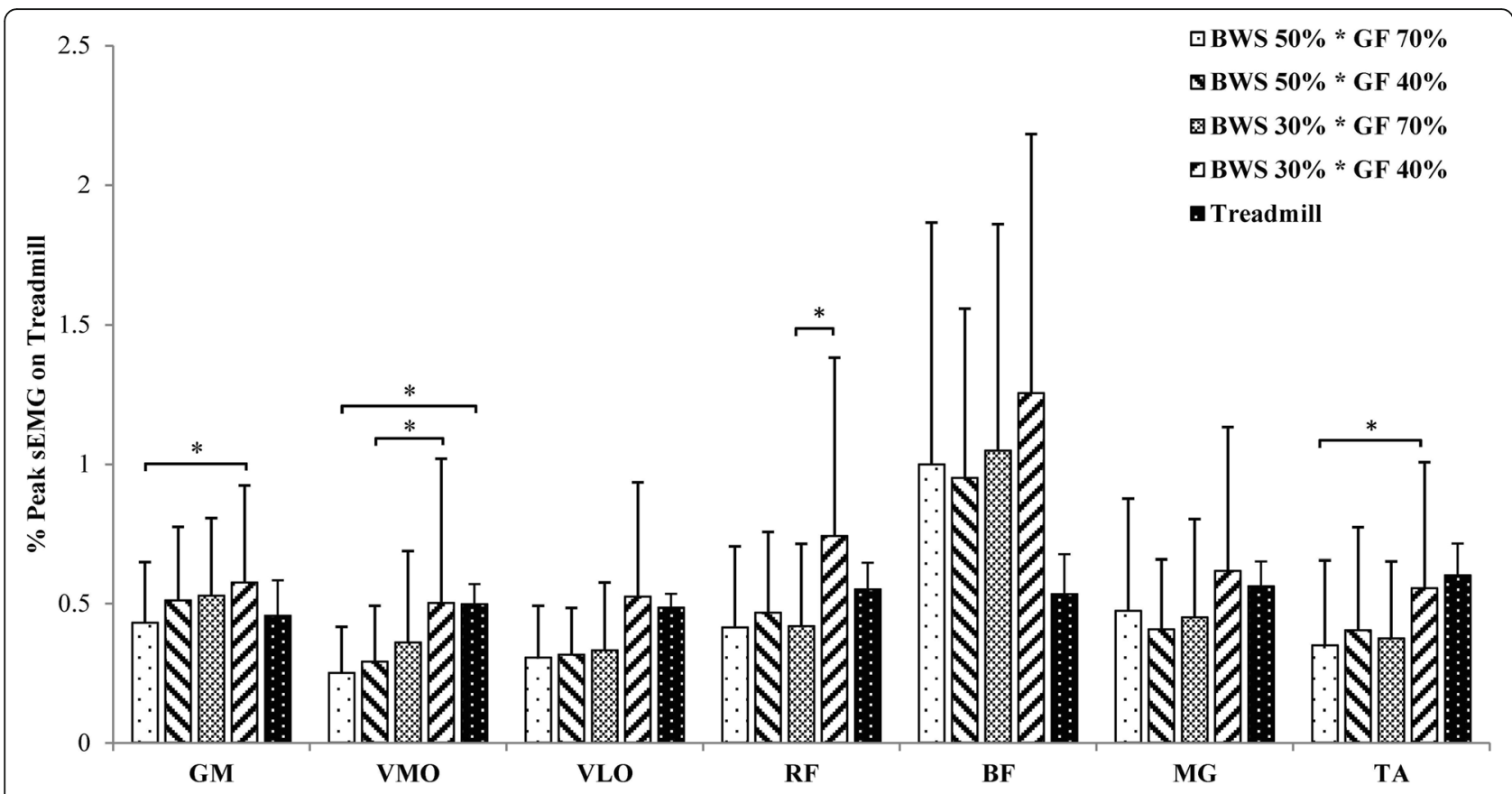

Fig. 3 Mean and standard deviation of normalised muscle activity amplitude. sEMG: surface electromyography; BWS: body weight support; GF: guidance force; GM: gluteus medius; VMO: vastus medialis oblique; VLO: vastus lateralis oblique; RF: rectus femoris; BF: biceps femoris; MG: medial gastrocnemius; TA: tibialis anterior

which can be confirmed by the interaction force between participants' leg and exoskeleton [10]. Therefore, greater muscle activity will be exerted when the exoskeletons resist the active leg movement. For example, in present study, the activity of BF during Locomat walking in the first half of gait cycle was relatively higher than that during treadmill walking. This may result from the interaction between the leg and exoskeleton when the knee movement was not compliant with the built-in gait pattern.

\section{Effects of varying BWS and GF on muscle activity during Locomat walking}

The present study has revealed that increasing BWS and GF could reduce muscle activity in specific muscles which are in light with some previous studies on healthy individuals [32, 42, 43]. However, in a recent research on stroke [34], authors reported that BWS and GF had little effect on muscle activity. The different results may not be directly related to the magnitude of change in BWS and GF as the magnitude of change in BWS and GF in our study were $20 \%$ (30 and $50 \%$ of body weight) and $30 \%$ (70 and $40 \%$ of guidance force) respectively, whereas that in previous study were $50 \%$ (0 and $50 \%$ of body weight) and $50 \%$ (50 and $100 \%$ of guidance force) respectively. But the magnitude of change in muscle activity may be related to the level of GF. van Kammen et al. [34] speculated that more voluntary muscle contraction may be stimulated if the guidance level is lower than $50 \%$. This speculation could be supported by the present study and other studies where one of the GF settings was lower than $50 \%$ and significant changes were reported [32, 33, 43]. This evidence indicates that there may be a threshold of GF under which the active muscle activities may increase during Locomat walking. Another potential explanation for the different findings may be that the participants in our study walked at a faster speed $(1.2 \mathrm{~m} / \mathrm{s})$ than theirs $(0.56 \mathrm{~m} / \mathrm{s})$ as higher walking speed can lead to greater muscle activity [34, 35, 38-41].

Moreover, the muscles affected by varying BWS and GF in current study were inconsistent with those in previous studies on healthy individuals. For example, in present study, reducing GF led to increased mean sEMG amplitude of RF while similar effects were reported on erector spinae, gluteus medius, biceps femoris, gluteus medius and tibialis anterior in previous studies on healthy individuals [32, 33, 43]. The different results of studies may attribute to the different muscle synergies, as the participants in these previous studies were neurologically intact while that in present study were with stroke.

The findings in this study may be limited by several factors. First, the participant gender is not balanced as only one female participant involved, which may introduce a bias. Second, participants in this study were at different courses post-stroke which made the results not generalizable to specific stroke population. There is compelling evidence showing that the most walking function recovered in the acute stage post-stroke [44-47]. Although the underlying mechanisms remain unclear, the 
recovery pattern of motor function post stroke suggests that course of post-stroke plays a significant role in recovery of motor function. In other words, the people with acute stroke may respond differently to the same Locomat setting when compared to people with chronic stroke. The future study should address the effects of course post-stroke by using specific stroke population and a longitudinal design. Third, the walking function of the participants was relatively good as they could walk unrestrained on a treadmill at a speed of $1.2 \mathrm{~m} / \mathrm{s}$. The results in this study may not apply to the participants with lower walking capacity. Fourth, the walking speed used in present study was relatively high for participant with stroke. The present findings may not reflect the effects of BWS and GF at a lower walking speed as there may be interactions between those parameters [32]. In addition, the temporal characteristics of muscle activation were not investigated in present study which made the effects of BWS and GF on the muscle activity level during each phase of gait cycle unknown. In order to demonstrate temporal characteristics of sEMG profile, a figure of mean sEMG amplitude over a gait cycle was provided to enable visual comparisons between different walking conditions. However, the gait cycle during Locomat guided walking is modulated by the build-in gait pattern and presents different duration of each phase compared to that during unrestrained treadmill walking. As aforementioned, the physical constraints by Locomat may lead to abnormal interaction between the legs and exoskeletons as well as abnormal muscle activity pattern $[10,22]$. Those factors may limit the significance of comparing temporal characteristics of muscle activity between Locomat guided walking and treadmill walking. Finally, the changes of muscle activity observed in present study were immediate effects rather than longterm effects. To better examine the effects of Locomat training on muscle activity, future research should measure both the spatial and temporal characteristics of muscle activity with long-term follow-ups.

The findings in present study may suggest that a normal walking speed should be selected for people with stroke during Locomat training. Locomat guided walking has been commonly reported to reduce muscle activity which is viewed as a negative aspect of its clinical implication because voluntary contraction of muscle plays a key role in motor relearning [21]. The findings in our study showed that when a normal walking speed was used for Locomat training, its effect on reducing muscle activity was minimized. The present study also suggested that clinicians could modulate the activities of specific muscles by adjusting BWS and GF in people with stroke. More specifically, if the training target is to reduce undesirable activities in GM, VMO, RF and TA, higher BWS and GF should be selected. On the other hand, if higher voluntary muscle activity is desired, then lower
BWS and GF should be employed. However, individuals may respond to the same Locomat training protocol differently. For example, the maximal muscle activity occurred at different combination of guidance force and body weight support in different patients [48]. Given that the interaction between active leg movement and exoskeleton could modulate the neuromuscular control, it would be of interest to clinical practice to investigate the role of the interaction between leg and exoskeleton in modulating muscle activity at each phase during a gait cycle, as well as its association with BWS, GF and speed.

\section{Conclusions}

The present study showed that the effects of Locomat on reducing muscle activity in people with stroke were minimized when walking at a normal speed. This study also revealed that reducing bodyweight support and guidance force increased the activity amplitude of specific muscle groups during Locomat walking. The findings of this study would suggest that effects of bodyweight support, guidance force and speed should be taken into account when developing individualized Locomat training protocols for clients with stroke.

\section{Abbreviations}

BF: Biceps femoris; BWS: Bodyweight support; GF: Guidance force; GM: Gluteus medius; MG: Medial gastrocnemius; RF: Rectus femoris; sEMG: Surface electromyography; TA: Tibialis anterior; VLO: Vastus lateralis oblique; VMO: Vastus medialis oblique

\section{Acknowledgements}

The authors thank all the participants in present study for their participation and valuable feedbacks.

\section{Authors' contributions}

$J H L$ designed the experiment, analysed and interpreted the data, and drafted the manuscript. GJH contributed to study design, participant recruitment, data collection and data processing. JR, LYC and XZ helped conducting the trials and improving test protocol. YXZ contributed to study design, data analysis and interpretation, as well as revision of manuscript. All authors read and approved the final manuscript.

\section{Funding}

The present study was funded by grant from Shanghai Disabled Persons' Federation (K2016029).

\section{Availability of data and materials}

The datasets used and/or analysed during the current study are available from the corresponding author on reasonable request.

\section{Ethics approval and consent to participate}

This study was approved by the Research Ethics Committee, Shanghai Yangzhi Affiliated Rehabilitation Hospital of Tongji University (YZ2016-032). Written informed consent was obtained from all participants.

Consent for publication

Not applicable.

\section{Competing interests}

The authors declare that they have no competing interests.

\section{Author details}

${ }^{1}$ Department of Rehabilitation Therapy, Yangzhi Affiliated Rehabilitation Hospital of Tongji University, No. 2209, Guangxing Road, Songjiang District, 
Shanghai 201619, People's Republic of China. ${ }^{2}$ Faculty of Health Sciences, The University of Sydney, Sydney, New South Wales, Australia. ${ }^{3}$ Department of Exercise Sciences, University of Auckland, Auckland, New Zealand.

Received: 9 July 2019 Accepted: 7 January 2020

Published online: 13 January 2020

\section{References}

1. Olney SJ, Richards C. Hemiparetic gait following stroke. Part I: Characteristics. Gait Posture. 1996;4(2):136-48.

2. Den Otter AR, Geurts AC, Mulder T, Duysens J. Abnormalities in the temporal patterning of lower extremity muscle activity in hemiparetic gait. Gait Posture. 2007;25(3):342-52

3. Go AS, Mozaffarian D, Roger VL, Benjamin EJ, Berry JD, Blaha MJ, et al. Executive summary: heart disease and stroke statistics--2014 update: a report from the American Heart Association. Circulation. 2014;129(3):399-410.

4. Forster A, Young J. Incidence and consequences of falls due to stroke: a systematic inquiry. BMJ. 1995;311(6997):83-6.

5. Batchelor FA, Mackintosh SF, Said CM, Hill KD. Falls after stroke. Int I Stroke. 2012;7(6):482-90.

6. Raffin E, Hummel FC. Restoring motor functions after stroke: multiple approaches and opportunities. Neuroscientist. 2018;24(4):400-16.

7. French B, Thomas LH, Coupe J, McMahon NE, Connell L, Harrison J, et al. Repetitive task training for improving functional ability after stroke. Cochrane Database Syst Rev. 2016;11:Cd006073.

8. Kwakkel G, van Peppen R, Wagenaar RC, Wood Dauphinee S, Richards C Ashburn A, et al. Effects of augmented exercise therapy time after stroke: a meta-analysis. Stroke. 2004;35(11):2529-39.

9. Hidler J, Nichols D, Pelliccio M, Brady K, Campbell DD, Kahn JH, et al. Multicenter randomized clinical trial evaluating the effectiveness of the Lokomat in subacute stroke. Neurorehabil Neural Repair. 2009;23(1):5-13.

10. Hidler JM, Wall AE. Alterations in muscle activation patterns during roboticassisted walking. Clin Biomech (Bristol, Avon). 2005;20(2):184-93.

11. Picelli A, Bacciga M, Melotti C, LAM E, Verzini E, Ferrari F, et al. Combined effects of robotassisted gait training and botulinum toxin type a on spastic equinus foot in patients with chronic stroke: a pilot, single blind, randomized controlled trial. Eur J Phys Rehabil Med. 2016;52(6):759-66.

12. Hesse $\mathrm{S}$, Waldner $\mathrm{A}$, Tomelleri $\mathrm{C}$. Innovative gait robot for the repetitive practice of floor walking and stair climbing up and down in stroke patients. J Neuroeng Rehabil. 2010;7:30.

13. Peurala SH, Airaksinen O, Huuskonen P, Jakala P, Juhakoski M, Sandell K, et al. Effects of intensive therapy using gait trainer or floor walking exercises early after stroke. J Rehabil Med. 2009:41(3):166-73.

14. Ng MF, Tong RK, Li LS. A pilot study of randomized clinical controlled trial of gait training in subacute stroke patients with partial body-weight support electromechanical gait trainer and functional electrical stimulation: sixmonth follow-up. Stroke. 2008:39(1):154-60.

15. Pohl M, Werner C, Holzgraefe M, Kroczek G, Mehrholz J, Wingendorf I, et al. Repetitive locomotor training and physiotherapy improve walking and basic activities of daily living after stroke: a single-blind, randomized multicentre trial (DEutsche GAngtrainerStudie, DEGAS). Clin Rehabil. 2007;21(1):17-27.

16. Marchal-Crespo L, Riener R. Chapter 16 - robot-assisted gait training. In: Colombo R, Sanguineti V, editors. Rehabilitation Robotics. London: Academic Press; 2018. p. 227-40.

17. Colombo G, Joerg M, Schreier R, Dietz V. Treadmill training of paraplegic patients using a robotic orthosis. J Rehabil Res Dev. 2000;37(6):693-700.

18. Riener R, Lünenburger L, Maier IC, Colombo G, Dietz V. Locomotor training in subjects with Sensori-motor deficits: an overview of the robotic gait Orthosis Lokomat. J Healthc Eng. 2010:1(2):197-216.

19. van Kammen K, Boonstra AM, van der Woude LHV, Reinders-Messelink HA den Otter R. Differences in muscle activity and temporal step parameters between Lokomat guided walking and treadmill walking in post-stroke hemiparetic patients and healthy walkers. J Neuroeng Rehabil. 2017:14(1):32

20. Coenen P, van Werven G, van Nunen MP, Van Dieen JH, Gerrits KH, Janssen TW. Robot-assisted walking vs overground walking in stroke patients: an evaluation of muscle activity. J Rehabil Med. 2012:44(4):331-7.

21. Kaelin-Lang A, Sawaki L, Cohen LG. Role of voluntary drive in encoding an elementary motor memory. J Neurophysiol. 2005;93(2):1099-103.

22. Neckel ND, Blonien N, Nichols D, Hidler J. Abnormal joint torque patterns exhibited by chronic stroke subjects while walking with a prescribed physiological gait pattern. J Neuroeng Rehabil. 2008;5:19.
23. Cho J-E, Yoo JS, Kim KE, Cho ST, Jang WS, Cho KH, et al. Systematic review of appropriate robotic intervention for gait function in subacute stroke patients. Biomed Res Int. 2018;2018:11.

24. Bruni MF, Melegari C, De Cola MC, Bramanti A, Bramanti P, Calabro RS. What does best evidence tell us about robotic gait rehabilitation in stroke patients: a systematic review and meta-analysis. J Clin Neurosci. 2018:48:11-7.

25. Mehrholz J, Thomas S, Werner C, Kugler J, Pohl M, Elsner B. Electromechanical-assisted training for walking after stroke. Cochrane Database Syst Rev. 2017;5:Cd006185.

26. Westlake KP, Patten C. Pilot study of Lokomat versus manual-assisted treadmill training for locomotor recovery post-stroke. J Neuroeng Rehabil. 2009:6(1):18.

27. Schwartz I, Sajin A, Fisher I, Neeb M, Shochina M, Katz-Leurer M, et al. The effectiveness of Locomotor therapy using robotic-assisted gait training in subacute stroke patients: a randomized controlled trial. PM\&R. 2009;1(6): $516-23$

28. Dundar U, Toktas H, Solak O, Ulasli AM, Eroglu S. A comparative study of conventional physiotherapy versus robotic training combined with physiotherapy in patients with stroke. Top Stroke Rehabil. 2014;21(6):453-61.

29. Hornby TG, Campbell DD, Kahn JH, Demott T, Moore JL, Roth HR. Enhanced gait-related improvements after therapist- versus robotic-assisted locomotor training in subjects with chronic stroke: a randomized controlled study. Stroke. 2008;39(6):1786-92.

30. Husemann B, Muller F, Krewer C, Heller S, Koenig E. Effects of locomotion training with assistance of a robot-driven gait orthosis in hemiparetic patients after stroke: a randomized controlled pilot study. Stroke. 2007;38(2): $349-54$.

31. van Nunen MP, Gerrits KH, Konijnenbelt M, Janssen TW, de Haan A. Recovery of walking ability using a robotic device in subacute stroke patients: a randomized controlled study. Disabil Rehabil Assist Technol. 2015;10(2):141-8.

32. van Kammen $\mathrm{K}$, Boonstra $\mathrm{AM}$, van der Woude $\mathrm{LH}$, Reinders-Messelink $H A$, den Otter R. The combined effects of guidance force, bodyweight support and gait speed on muscle activity during able-bodied walking in the Lokomat. Clin Biomech (Bristol, Avon). 2016;36:65-73.

33. Moreno JC, Barroso F, Farina D, Gizzi L, Santos C, Molinari M, et al. Effects of robotic guidance on the coordination of locomotion. J Neuroeng Rehabil. 2013;10:79.

34. van Kammen K, Boonstra AM, van der Woude LHV, Visscher C, ReindersMesselink HA, den Otter R. Lokomat guided gait in hemiparetic stroke patients: the effects of training parametters on muscle activity and tempora symmetry. Disabil Rehabil [Journal on the Internet]. 2019 Apr 11 [cited 2019 Jul 9]. Available from: https://doi.org/10.1080/09638288.2019.1579259. [Epub ahead of print].

35. Burnfield JM, Buster TW, Goldman AJ, Corbridge LM, Harper-Hanigan K. Partial body weight support treadmill training speed influences paretic and nonparetic leg muscle activation, stride characteristics, and ratings of perceived exertion during acute stroke rehabilitation. Hum Mov Sci. 2016;47:16-28.

36. Konrad P, The ABC. Of EMG. A practical introduction to Kinesiological electromyography. Scottsdale: Noraxon U.S.A. Inc; 2006.

37. Freriks B, Hermens H, Disselhorst-Klug C, Rau G. The Recommendations for Sensors and Sensor Placement Procedures for Surface ElectroMyoGraphy. In: Hermens $\mathrm{H}$, editor. European Recommendations for Surface Electromyography: Results of the SENIAM Project: Roessingh Research and Development bv; 1999. p. 15-53.

38. Lamontagne A, Fung J. Faster is better: implications for speed-intensive gait training after stroke. Stroke. 2004;35(11):2543-8.

39. den Otter AR, Geurts AC, Mulder T, Duysens J. Speed related changes in muscle activity from normal to very slow walking speeds. Gait Posture. 2004;19(3):270-8.

40. Ivanenko YP, Grasso R, Macellari V, Lacquaniti F. Control of foot trajectory in human locomotion: role of ground contact forces in simulated reduced gravity. J Neurophysiol. 2002;87(6):3070-89.

41. Hof AL, Elzinga H, Grimmius W, Halbertsma JP. Speed dependence of averaged EMG profiles in walking. Gait Posture. 2002;16(1):78-86.

42. Van Kammen K, Boonstra A, Reinders-Messelink H, den Otter R. The combined effects of body weight support and gait speed on gait related muscle activity: a comparison between walking in the Lokomat exoskeleton and regular treadmill walking. PLoS One. 2014;9(9):e107323.

43. Weiland S, Smit IH, Reinders-Messelink H, van der Woude LHV, van Kammen $K$, den Otter R. The effect of asymmetric movement support on muscle 
activity during Lokomat guided gait in able-bodied individuals. PLoS One. 2018;13(6):e0198473.

44. Askim T, Bernhardt J, Salvesen O, Indredavik B. Physical activity early after stroke and its association to functional outcome 3 months later. J Stroke Cerebrovasc Dis. 2014;23(5):e305-12.

45. Kollen B, van de Port I, Lindeman E, Twisk J, Kwakkel G. Predicting improvement in gait after stroke: a longitudinal prospective study. Stroke. 2005;36(12):2676-80.

46. Horn SD, DeJong G, Smout RJ, Gassaway J, James R, Conroy B. Stroke rehabilitation patients, practice, and outcomes: is earlier and more aggressive therapy better? Arch Phys Med Rehabil. 2005;86(12 Suppl 2): S101-s14.

47. Jorgensen HS, Nakayama H, Raaschou HO, Olsen TS. Recovery of walking function in stroke patients: the Copenhagen stroke study. Arch Phys Med Rehabil. 1995:76(1):27-32

48. Cherni Y, Begon M, Chababe H, Moissenet F. Use of electromyography to optimize Lokomat ${ }^{\oplus}$ settings for subject-specific gait rehabilitation in poststroke hemiparetic patients: a proof-of-concept study. Neurophysiol Clin. 2017:47(4):293-9.

\section{Publisher's Note}

Springer Nature remains neutral with regard to jurisdictional claims in published maps and institutional affiliations.

Ready to submit your research? Choose BMC and benefit from:

- fast, convenient online submission

- thorough peer review by experienced researchers in your field

- rapid publication on acceptance

- support for research data, including large and complex data types

- gold Open Access which fosters wider collaboration and increased citations

- maximum visibility for your research: over $100 \mathrm{M}$ website views per year

At BMC, research is always in progress.

Learn more biomedcentral.com/submissions 